\title{
Graves' ophthalmopathy: II. Correlation of clinical signs with measures derived from computed tomography
}

\author{
E SVERKER HALLIN' AND STEVEN E FELDON ${ }^{2}$
}

\begin{abstract}
From the 'Department of Ophthalmology, University of Göteborg, Sweden, and the ${ }^{2}$ Departments of Ophthalmology and Neurological Surgery, University of Southern California School of Medicine, Los Angeles, California, USA
\end{abstract}

SUMmaRY Quantitative clinical and computed tomographic (CT) measures of Graves' ophthalmopathy were obtained in 76 patients. Significant correlation of several CT parameters of the mid-orbital axial CT slice with optic nerve involvement was established. By means of clinical measures, only limitation of ocular motility was found to correlate with the optic neuropathy. The results suggested that there were two distinct populations of patients with Graves' disease which could be segregated by CT scan and clinical evaluation, one which developed optic neuropathy and one which did not. Therefore simple measures performed on the mid-orbital slice of the CT scan are recommended for all patients with Graves' ophthalmopathy as an important adjunct to careful evaluation of extraocular muscle function, in order to identify patients at risk of developing optic neuropathy.

Computed tomography is a useful technique in the assessment of Graves' ophthalmopathy. ${ }^{1-4}$ When used in association with limitation of extraocular muscle function, computation of total extraocular muscle volume on CT identifies the population at risk for developing optic neuropathy..$^{5-7}$ However, several quantitative measures other than total extraocular muscle volume have now been shown to be highly correlated with total volume. These new measures are much easier and faster to obtain by either automatic or manual techniques of analysis. ${ }^{8}$ The purpose of this paper is to correlate these other CTderived measures with clinical manifestations of Graves' ophthalmopathy. In so doing, an optimal technique for assessing patients with Graves' ophthalmopathy can be established.

\section{Material and methods}

One hundred and fifty-two orbits in 76 patients with Graves' ophthalmopathy were studied and have been described in a companion paper. ${ }^{8}$ An analysis of CT scans was performed, consisting of the quantitative Correspondence to E Sverker Hallin, MD, Ogonkliniken, Sahlgrenska sjukhuset, S-413 45 Göteborg, Sweden. estimation of extraocular muscle volume, mid-orbital extraocular muscle area (the area of medial and lateral recti being added together), mid-orbital medial rectus muscle area, and width (Fig. 1). Furthermore, on the mid-orbital axial CT slice (defined as that section imaging the crystalline lens, the optic nerve head, and the optic canal) preseptal area, proptosis, and the ratio of orbit to fat were calculated. The derivation of these CT measures has been described in detail elsewhere. ${ }^{8}$

In addition to CT analysis the following clinical signs of Graves' ophthalmopathy were evaluated: proptosis, periorbital oedema, lid retraction, lid lag, limitation of ocular motility, and optic neuropathy. Proptosis was measured with the Krahn exophthalmometer. Periorbital oedema was assessed in 112 of the orbits by measuring herniation of the upper lid tissue over the upper lid fold. Lid retraction was established as the exposure of sclera superior to the corneal limbus. Lid lag was measured in a manner similar to lid retraction, but with the eyes directed downwards (Fig. 2). Limitation of ocular motility was calculated as a percentage of full excursion in both horizontal and vertical directions of gaze. The semiquantitative techniques used to determine the clinical 


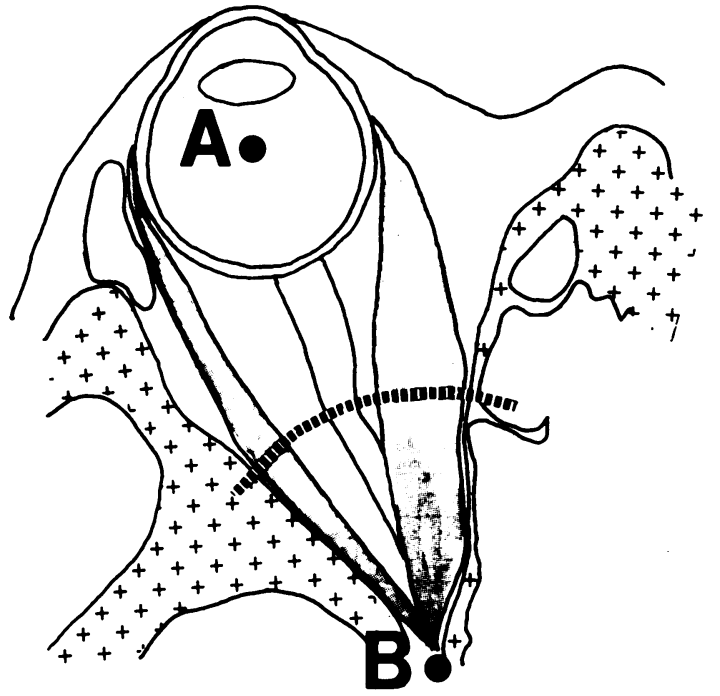

Fig. 1 Camera lucida drawing of the mid-orbital axial slice of a CT scan. Point $A$ is the centre of the globe. The dashed arcline is equidistant from the orbital apex $(\mathrm{B})$ including the midpoint of AB. Along the arc medial muscle width and orbit-fat ratio were calculated.

signs of Graves' ophthalmopathy have been described in detail elsewhere. ${ }^{56}$

Optic neuropathy was defined by the signs of optic nerve head swelling or atrophy, the presence of afferent pupillary defect, the loss of acuity or colour vision, or the presence of visual field defects not attributable to other eye disease. Patients were categorised as having no optic neuropathy (group 1, 50 patients), having developed optic neuropathy during follow-up (group 2, 13 patients), or having presented with optic neuropathy (group 3, 13 patients). The minimum follow-up time was six

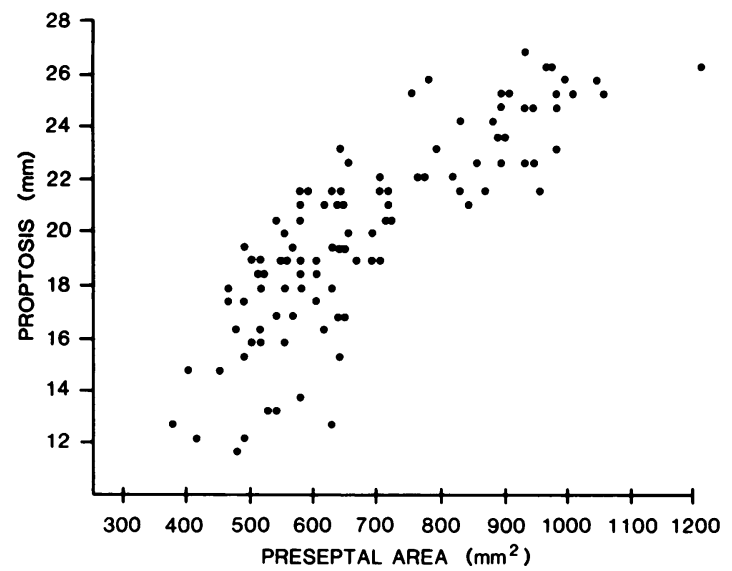

Fig. 3 Scatterplot demonstrating the relation between proptosis and preseptal area both measured by quantitative CT analysis. Proptosis shows a linear increase with preseptal area only through about $70 \%$ of its full range.
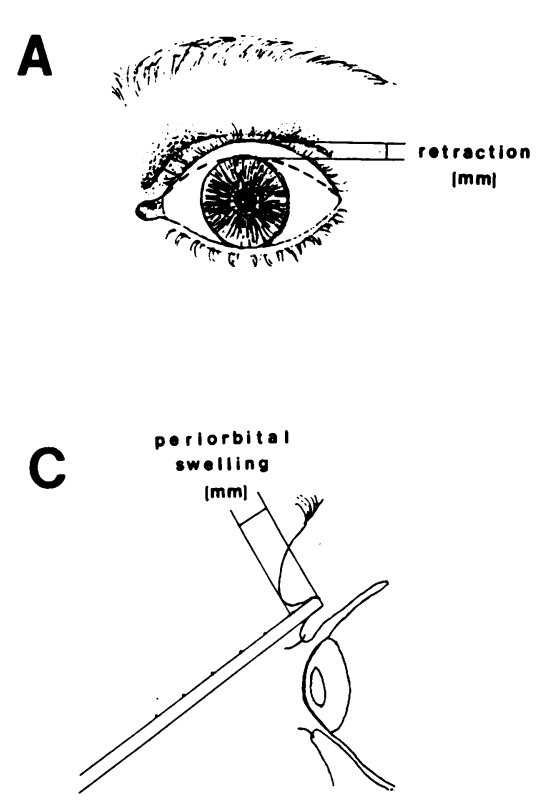
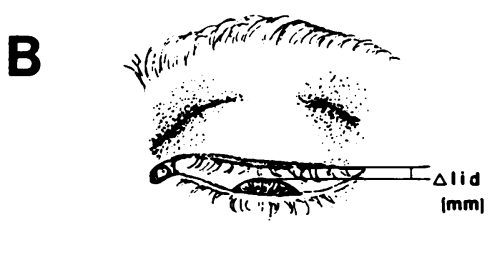

\section{D}

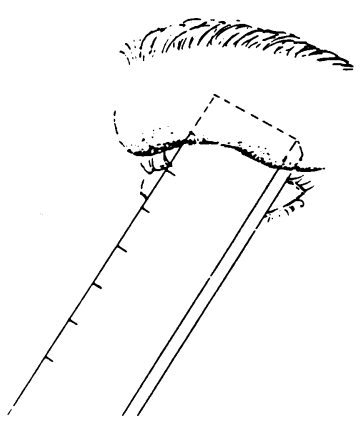

Fig. 2 Semiquantitative techniques for evaluating clinical signs of Graves' ophthalmopathy are shown. A: The distance between the superior limbus and lid with eye in primary position of gaze is lid retraction. B: This distance measured in downgaze is lid lag. C, D: The distance between the lid fold and the anterior extension of the periorbital tissue of the upper lid is the measure of periorbital oedema. 
Table 1 Correlation of clinical and CT signs of Graves' ophthalmopathy

\begin{tabular}{|c|c|c|c|c|c|c|}
\hline & $\begin{array}{l}\text { Optic nerve } \\
\text { involvement ( } p)\end{array}$ & $\begin{array}{l}\text { Motility limitation } \\
(p)\end{array}$ & $\begin{array}{l}\text { Periorbital oedema } \\
\text { (p) }\end{array}$ & $\begin{array}{l}\text { Exophthalmometry } \\
(p)\end{array}$ & Lid retraction $(p)$ & Liglag $(p)$ \\
\hline \multicolumn{7}{|l|}{ CT measurements } \\
\hline Muscle area & $0.65(\mathrm{p}<0.001)^{*}$ & $0.40(p<0.001)$ & $0.46(p<0.001)$ & $0.39(p<0.001)$ & $0 \cdot 10(\mathrm{NS})$ & $0 \cdot 00(\mathrm{NS})$ \\
\hline Total muscle volume & $0.65(\mathrm{p}<0.001)$ & $0.46(p<0.001)$ & $0.43(\mathrm{p}<0.001)$ & $0.43(\mathrm{p}<0.001)$ & $0 \cdot 15$ (NS) & 0.01 (NS) \\
\hline Medial rectus width & $0.61(\mathrm{p}<0.001)$ & $0.55(\mathrm{p}<0.001)$ & $0.44(p<0.001)$ & $0.29(p<0.01)$ & $0 \cdot 11$ (NS) & 0.07 (NS) \\
\hline Medial muscle area & $0.57(p<0.001)$ & $0.51(\mathrm{p}<0.001)$ & $0.49(\mathrm{p}<0.001)$ & $0.45(\mathrm{p}<0.001)$ & $0 \cdot 17$ (NS) & 0.03 (NS) \\
\hline Orbit/fat ratio & $0.54(\mathrm{p}<0.001)$ & $0.44(\mathrm{p}<0.001)$ & $0.28(\mathrm{NS})$ & $0 \cdot 18(\mathrm{NS})$ & $0 \cdot 10$ (NS) & 0.01 (NS) \\
\hline Preseptal area & $0.42(p<0.001)$ & $0.36(\mathrm{p}<0.001)$ & $0.47(\mathrm{p}<0.001)$ & $0.57(\mathrm{p}<0.001)$ & $0 \cdot 16(\mathrm{NS})$ & $0 \cdot 14(\mathrm{NS})$ \\
\hline Proptosis & $0.28(\mathrm{p}<0.02)$ & $0.26(\mathrm{p}<0.02)$ & $0.37(\mathrm{p}<0.01)$ & $0.73(\mathrm{p}<0.001)$ & $0 \cdot 16(\mathrm{NS})$ & $0 \cdot 13$ (NS) \\
\hline \multicolumn{7}{|l|}{ Clinical signs } \\
\hline Motility limitation & $0.45(p<0.001)$ & - & $0.32(\mathrm{p}<0.02)$ & $0 \cdot 18(\mathrm{NS})$ & $0 \cdot 21(\mathrm{NS})$ & $0 \cdot 09$ (NS) \\
\hline Periorbital oedema & $0.35(\mathrm{p}<0.01)$ & $0.32(\mathrm{p}<0.02)$ & - & $0 \cdot 29$ (NS) & $0.06(\mathrm{NS})$ & $0 \cdot 00$ (NS) \\
\hline Exophthalmometry & $0 \cdot 14$ (NS) & $0 \cdot 18(\mathrm{NS})$ & $0 \cdot 29$ (NS) & - & $0 \cdot 10(\mathrm{NS})$ & $0 \cdot 01$ (NS) \\
\hline Lid lag & $0.06(\mathrm{NS})$ & 0.09 (NS) & 0.00 (NS) & $0 \cdot 01$ (NS) & $0.30(\mathrm{p}<0.01)$ & - \\
\hline Lid retraction & 0.00 (NS) & $0 \cdot 21$ (NS) & 0.06 (NS) & $0 \cdot 10(\mathrm{NS})$ & - & $0.30(\mathrm{p}<0.01)$ \\
\hline
\end{tabular}

${ }^{*} p$ Values $>0.001$ were not considered as significant because multiple correlation was performed.

months. The group number was used as a continuous variable in estimating the degree of optic nerve involvement for the purpose of calculating correlations with other measures of Graves' ophthalmopathy.

\section{Results}

\section{CORRELATION OF CLINICAL SIGNS WITH CT MEASUREMENTS}

Clinical signs of Graves' ophthalmopathy were correlated with several quantitative measures determined from the CT scans. These results are summarised in Table 1. As expected, total extraocular muscle volume was highly correlated with the presence of optic neuropathy $(r=0.65, p<0.001)$ and was also significantly correlated with proptosis, total extraocular muscle limitation, and orbital swelling. Similar high correlations with optic neuropathy were also obtained using either the muscle area $(r=0.65$, $p<0.001)$ or the medial rectus muscle area ( $r=0.57$, $\mathrm{p}<0.001)$. Interestingly, medial rectus muscle width showed the highest correlation of any CT measure with total limitation of extraocular muscle function $(r=0.55, p<0.001)$, and was nearly as highly correlated as the more complex volume and area measures of the extraocular muscles with optic neuropathy $(r=0.61, p<0.001)$ and periorbital oedema $(r=0.44$, $\mathrm{p}<0.001)$.

\section{CORRELATION OF CLINICAL SIGNS WITH EACH OTHER}

Clinical signs of Graves' ophthalmopathy were correlated with each other. The results are summarised in Table 1 . Significant correlations were obtained only for clinical measures compared with the occurrence of optic neuropathy. Even so, total limitation of extraocular motility $(\mathrm{r}=0.45, \mathrm{p}<0.001)$ and periorbital oedema $(r=0.35, p<0.01)$ were less correlated with optic neuropathy than were many of the CT measures.

\section{EXOPHTHALMOMETRY AND PERIORBITAL} OEDEMA

Exophthalmometry, a frequently used but highly variable method $^{910}$ for quantitation of Graves' ophthalmopathy, showed poor correlation with optic neuropathy $(r=0 \cdot 14, p>0 \cdot 1)$ and other clinical signs. Proptosis determined from the CT scans correlated significantly with neither optic neuropathy nor total limitation of ocular motility, but did correlate highly with Krahn exophthalmometry $(r=0 \cdot 73)$. The preseptal area was defined as all tissue anterior to a line .connecting the lateral orbital rims, limited by the soft tissue laterally and orbital wall medially. The preseptal area encompassed elements of both periorbital oedema and proptosis, and it was significantly correlated with optic neuropathy and other clinical signs, such as total limitation of motility and periorbital oedema.

A scatterplot comparing preseptal area with proptosis showed a linear relationship through about $70 \%$ of the total range of proptosis. After proptosis was maximal, preseptal area continued to increase. Therefore, preseptal area was a more effective quantitative measure of orbital swelling in Graves' ophthalmopathy than were Krahn or Hertel readings (Fig.3).

MEASURES IDENTIFYING PATIENTS AT RISK FOR OPTIC NEUROPATHY

An attempt was made further to compare the ability of various CT and clinical measures to confirm the presence or predict the development of optic neuro- 
Table 2 Evaluation of clinical and CT signs of Graves' ophthalmopathy in separating patients with and without optic neuropathy

\begin{tabular}{|c|c|c|c|c|c|c|}
\hline & \multicolumn{2}{|c|}{ No risk optic vs present neuropathy } & \multicolumn{2}{|c|}{ No risk optic vs developed neuropathy } & \multicolumn{2}{|c|}{ Present optic vs developed neuropathy } \\
\hline & $t$ & $p$ & $t$ & $p$ & $t$ & $p$ \\
\hline \multicolumn{7}{|l|}{ CT measurements } \\
\hline Muscle area & $9 \cdot 88$ & $\mathrm{p}<0.0001^{*}$ & $6 \cdot 33$ & $p<0.0001$ & $2 \cdot 64$ & $\mathrm{p}<0.006$ \\
\hline Total muscle volume & $9 \cdot 68$ & $\mathrm{p}<0.0001$ & $6 \cdot 74$ & $\mathrm{p}<0.0001$ & $2 \cdot 50$ & $p<0.008$ \\
\hline Medial rectus width & $8 \cdot 93$ & $\mathrm{p}<0.0001$ & $6 \cdot 79$ & $\mathrm{p}<0.0001$ & $1 \cdot 39$ & NS $(p<0 \cdot 09)$ \\
\hline Medial muscle area & $7 \cdot 87$ & $\mathrm{p}<0.0001$ & $5 \cdot 66$ & $\mathrm{p}<0.0001$ & $1 \cdot 58$ & $N S(p<0 \cdot 07)$ \\
\hline Orbit/fat ratio & $7 \cdot 33$ & $\mathrm{p}<0.0001$ & $5 \cdot 84$ & $p<0.0001$ & $1 \cdot 06$ & $N S(p<0 \cdot 2)$ \\
\hline Preseptal area & $4 \cdot 37$ & $\mathrm{p}<0.0001$ & $4 \cdot 32$ & $p<0.0001$ & $0 \cdot 34$ & NS $(p<0.4)$ \\
\hline Proptosis & $3 \cdot 23$ & $\mathrm{p}<0.0001$ & $2 \cdot 92$ & $\mathrm{p}<0.003$ & 0.13 & $\mathrm{NS}(\mathrm{p}<0.5)$ \\
\hline \multicolumn{7}{|l|}{ Clinical signs } \\
\hline Motility limitation & $4 \cdot 49$ & $\mathrm{p}<0.0001$ & $5 \cdot 68$ & $\mathrm{p}<0.0001$ & $0 \cdot 88$ & $N S(p<0 \cdot 2)$ \\
\hline Periorbital oedema & 3.59 & $\mathrm{p}<0.0001$ & $3 \cdot 33$ & $\mathrm{p}<0.0001$ & $0 \cdot 26$ & $N S(p<0.4)$ \\
\hline Exophthalmometry & $1 \cdot 52$ & NS $(p<0.07)$ & $1 \cdot 21$ & $\mathrm{NS}(\mathrm{p}<0 \cdot 2)$ & 0.25 & $N S(p<0.5)$ \\
\hline Lid lag & $0 \cdot 72$ & $N S(p<0.3)$ & $0 \cdot 39$ & $N S(p<0.4)$ & $0 \cdot 17$ & $N S(p<0.4)$ \\
\hline Lid retraction & $0 \cdot 21$ & $N S(p<0.5)$ & 0.49 & $N S(p<0.4)$ & 0.52 & $N S(p<0.4)$ \\
\hline
\end{tabular}

${ }^{*} \mathrm{p}$ Values $>0.001$ were not considered as significant becaust multiple correlation was performed.

pathy. These comparisons were performed using multiple $t$ tests. Therefore, only $\mathrm{p}$ values less than 0.001 were considered statistically significant. These data are summarised in Table 2. Virtually all CT measures, including muscle width, could significantly $(p<0.0001)$ distinguish the groups that either had or developed optic neuropathy from the group that did not have optic neuropathy. The only clinical measures that distinguished these groups were total limitation of extraocular motility and periorbital oedema.

Neither CT measures nor clinical measures of Graves' ophthalmopathy successfully differentiated between the group that presented with optic neuropathy and the group that developed optic neuropathy. Some nearly significant differences were detectable between these groups by CT measures of extraocular muscle area $(\mathrm{p}<0.006)$ or volume $(\mathrm{p}<0.008)$.

\section{Discussion}

Clinical and CT measures of Graves' ophthalmopathy have been compared, especially with regard to their relationship to optic neuropathy. All CT measures of extraocular muscle enlargement correlated significantly; muscle area and total muscle volume were the most highly correlated. The only clinical measures that correlated significantly with optic neuropathy were limitation of ocular motility and perhaps periorbital oedema, confirming a prior study. ${ }^{6}$

Along with other CT-derived measures, medial rectus width, which may be obtained manually, was reliably able to distinguish between the group that would develop optic neuropathy and the group that would not. There seemed to be no reliable way of distinguishing the group that developed optic neuropathy from the group that presented with optic neuropathy. Therefore it is likely that these groups were in fact the same population.

This study has established quantitative CT evaluation of the extraocular muscles and preseptal area as the most important parameters in assessing and following up patients with Graves' ophthalmopathy. Muscle area can be determined by the radiologist using morphometric analysis available on most CT scanners. Similarly, preseptal area is easy to calculate and is a better measure of anterior manifestations of Graves' ophthalmopathy than are either Krahn exophthalmometry or clinical evaluation of periorbital oedema. If automatic analysis is not available, medial rectus muscle width is an exceptionally easy measure to obtain manually for evaluating risk of optic neuropathy, requiring only a single mid-orbital thin CT section and no special equipment, training, or calculation.

Although many clinical signs are of value in diagnosing orbital involvement with Graves' disease, limitation of motility and optic nerve changes only need be regularly evaluated once a baseline orbital CT scan has been performed and measured.

This study was supported in part by Handlande Herman Svenssons Fond, by De Blindas Vanner and by grant EY03908 awarded by the National Institutes of Health, USA.

ESH was a research fellow in the Department of Ophthalmology, University of Southern California School of Medicine.

\section{References}

1 Trokel SL, Hilal SK. Submillimeter resolution CT scanning of orbital diseases. Ophthalmology 1980; 387: 412-7. 
2 Kennerdell JS, Rosenbaum AE, El-Hoshy MH. Apical optic nerve compression of dysthyroid optic neuropathy on computed tomography. Arch Ophthalmol 1981; 99: 807-9.

3 Trokel SL, Jakobiec FA. Correlation of CT scanning and pathologic features of ophthalmic Graves' disease. Ophthalmology 1981; 88: 553-64.

4 Hallin S. Distribution of extraocular muscle swelling in endocrine exophthalmos. Prov VII Cong Eur Ophthalmol (Helsinki) 1985: 528-9.

5 Feldon SE, Weiner JM. Clinical significance of extraocular muscle volumes in Graves' ophthalmopathy: A quantitative computed tomography study. Arch Ophthalmol 1982; 100: 1266-9.

6 Feldon SE, Muramatsu S, Weiner JM. Clinical classification of Graves' ophthalmopathy: identification of risk factors for optic neuropathy. Arch Ophthalmol 1984; 102: 1469-72.
7 Feldon SE, Lee CP, Muramatsu SK, Weiner JM. Quantitative computed tomography of Graves' ophthalmopathy: extraocular muscle and orbital fat in development of optic neuropathy. Arch Ophthalmol 1985; 103: 213-5.

8 Hallin ES, Feldon SE. Graves' ophthalmopathy: I. Simple CT estimates of extraocular muscle volume. Br J Ophthalmol 1988 . 72: 674-7.

9 Rundle FF. Some observations on exophthalmos. West J Surg Obstet Gynecol 1947; 55: 578-83.

10 Amino N, Yuasa T, Yabu Y, Miyai K. Kumahara Y. Exophthalmos in autoimmune thyroid disease. J Clin Endocrinol $1980 ; 51: 1232-4$.

Accepted for publication 2 July 1987. 